\title{
Study on Management of Project for HK and Macao Exchange Program of Yunnan University of Finance and Economics under SECI Model
}

\author{
Mingshan Jiang* \\ International Cooperation and Exchange Office \\ Yunnan University of Finance and Economics \\ Kunming, Yunnan, China \\ School of Knowledge Science \\ Japan Advanced Institute of Science and Technology \\ Nomi, Ishikawa, Japan \\ prajnapeace@qq.com
}

\author{
Hongmin Li \\ International Cooperation and Exchange Office \\ Yunnan University of Finance and Economics \\ Kunming, Yunnan, China \\ 99663759@qq.com \\ Zhifei Li \\ International Cooperation and Exchange Office \\ Yunnan University of Finance and Economics \\ Kunming, Yunnan, China \\ lizhifei1115@126.com
}

\begin{abstract}
Through the concept of "Urban Agglomeration at Guangdong-HK-Macau Great Bay", as important members of "Guangdong-HK-Macau Bay Area", the educational exchange among HK, Macau and mainland have also aroused lots of attentions. Yunnan University of Finance and Economics joined the "College teachers and students Exchange program between Mainland and Hong Kong \& Macao" since 2012. This article aims at analyzing the strategies for potential maneuverability improvement during the management service under the guidance of SECI model according to the development features of knowledge in management service of "Exchange Program" to improve the management mechanism and service platform.
\end{abstract}

Keywords-the Belt and Road; HK \& Macao; exchange program; SECI model; knowledge management; innovative management

\section{INTRODUCTION}

"The belt and road" initiative has already evolved into an important pathway to strengthen overseas cooperation since it was proposed by President $\mathrm{Xi}$ Jinping during his visit to the Southeast Asia. The principle of joint discussion, construction and sharing based on which "the belt and road" is constructed is also developing in $\mathrm{HK}$ and Macao. The proposal of the development plan of "Urban Agglomeration at GuangdongHK-Macao Great Bay" at two sessions in 2017 highlights another emphasis on the regional cooperation. Through introduction of the concept of "bay area", new vigor is brought into the construction of "the belt and road". As important members of "Guangdong-HK-Macao Bay Area", the educational exchange among $\mathrm{HK}$, Macao and mainland have also aroused lots of attentions.

The project of "College teachers and students Exchange program between Mainland and Hong Kong \& Macao" (hereinafter referred to as "Exchange Program") is an important project platform that the central government set for the first time to support HK college teachers and students to experience and learn about scientific research in mainland at the 15th anniversary marking the return of HK in 2012. Macao colleges joined the platform in 2015 and shared policy dividends issued by the central government with HK. Yunnan University of Finance and Economics (hereinafter referred to as YUFE) was among the first batch of universities joining the program in 2012 to vigorously propel the exchanges of students from HK and Macao. Through analysis on problems occurring in the implementation and management of "Exchange Program", it is found that there is still space for making progress in its management service mechanism and construction of interconnection \& communication platform. It is aimed at analyzing the strategies for potential maneuverability improvement during the management service under the guidance of SECI model according to the development features of knowledge in management service of "Exchange Program" to improve the management mechanism and service platform.

\section{THEORETICAL BASIS OF THE RESEARCH}

Among dimensions of cognitive theory, Michael Polanyi [1] divides knowledge into explicit and tacit knowledge, the former indicates that the knowledge can be expressed with objective language and figure equation, shared through formula and handbook and spread directly formally and systematically among individuals while the latter indicates subjective inspiration \& intuition and the knowledge that is hard to be explicated and spread \& shared [2]. Polanyi lays emphasis on importance of tacit knowledge, pointing out that what we know is usually more than what we can say.

\footnotetext{
*Corresponding author
} 
In the changing era, knowledge innovation has undoubtedly been playing a very important role in organizational management. Ikujiro Nonaka, "the father of knowledge-creation" [3] believes that knowledge innovation originates from the interaction and transformation between explicit and tacit knowledge and Hirotaka Takeuchi proposes the SECIO model thereupon, revealing the law in knowledge creation. The SECI model is initially the classical theory created with knowledge. Its theory integrates the philosophy of the west and east, constructing a generally-applied knowledge management theoretical system with the characteristics of the east and the west. It comprehensively describes the four models of knowledge transformation and knowledge field $(\mathrm{Ba})$ of knowledge innovation. By virtue of its outstanding role in elaborating individual and organizational knowledge and realizing organization's goal, it has been applied into the management field. The paper aims at discussing the transformation of tacit knowledge to the explicit knowledge in organizational management of "Exchange Program" drawing upon SECI model in knowledge-creation theory to improve management strategy for "Exchange Program". Firstly, the basic interpretation of the SECI model is as below:

There is a basic premise for the SECI model. Knowledge innovation is realized and completed in group and situation of socializing. However, during implementation of the project of "Exchange Program", new strategies could be tapped based on experience and feedback of groups. In SECI model, the interaction of "Tacit knowledge" and "Explicit Knowledge" is concluded into four processes by combining the expressions of Ikujiro Nonaka [4]:

\section{A. Socialization}

The process of socialization, namely the tacit process which indicates the stage of conveying tacit knowledge through individual and realizing group sharing of tacit knowledge and changing personal experience into group sharing experience.

\section{B. Externalization}

This stage indicates the transformation of tacit knowledge into explicit knowledge. It is the process of expressing the tacit knowledge with symbolic concept and language to realize explicitness of knowledge. At this stage, using metaphorical language and symbolism can help express people's intuition and inspiration, thus being an effective pathway of "external explicitness".

\section{Combination}

Namely the process of summary and combination, indicating the combination and systematization of different knowledge concepts through various ways and transforming scattered explicit knowledge into systematic knowledge. This stage only represents the completion of explicit knowledge combination. Individual can obtain new knowledge fragment but the organization doesn't expend its existing knowledge reserve.

\section{Internalization}

Through internal sublimation of internalization, the explicit knowledge is transformed into tacit knowledge and the knowledge reserve of the organization is finally transformed into the personal knowledge creation. The new knowledge becomes personal and new tacit knowledge after being absorbed and sublimated by the organization's staff.

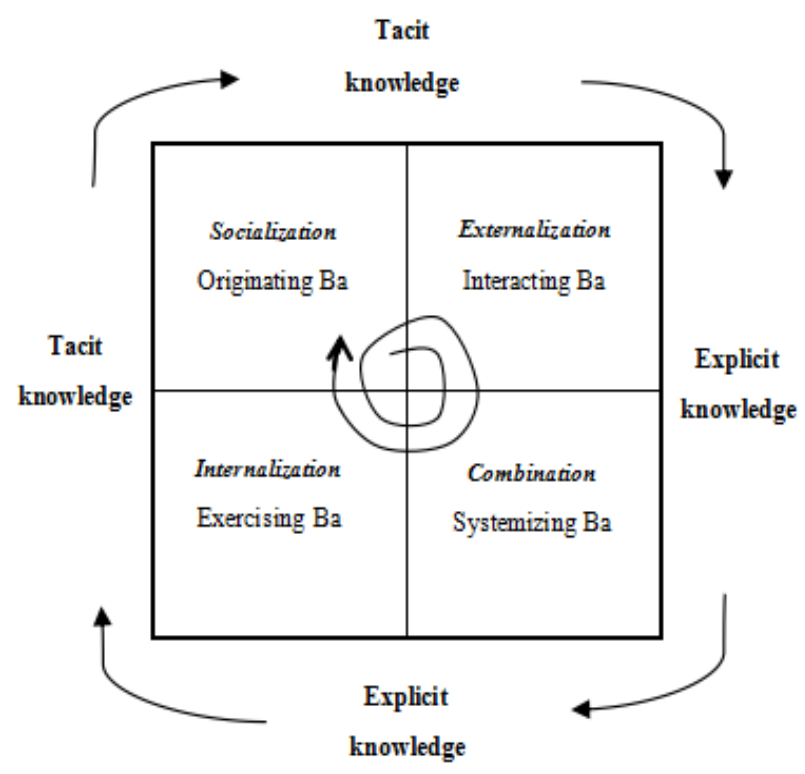

Fig. 1. SCEI Model Concept Diagram proposed by Ikujiro Nonaka and Hirotaka Takeuch

As shown in Fig. I, the transformation of four processes is completed in SECI model, namely completing a spiral dimension once. There exists a "field $(\mathrm{Ba})$ " at every process, namely Originating $\mathrm{Ba}$, Interacting $\mathrm{Ba}$, Systemizing $\mathrm{Ba}$ and Exercising $\mathrm{Ba}$. One base is provided for each bar respectively to handle the procedure of knowledge transformation and accelerate knowledge innovation. The final result of SECI model is to create new knowledge and form valuable opinions for the organizational management. The new round of knowledge creation process can start through a complete fourstage process.

\section{THE APPLICATION OF SECI MODEL INTO THE CASE OF "EXCHANGE PROGRAM"}

Apr. 2018, YUFE has successfully received 35 batches of student and teacher exchange groups from many universities such as HK University, City University of HK, HK Baptist University, HKPU, Education University of Hong Kong, Macao Higher Education Office and Macao International Cultural Education Exchange Association, 811 persons in total The number was increased to 218 in 2017 from 105 in 2013, featuring stable increase and remarkable achievement. During this period, the organizational goal was completed through conscious or unconscious, direct or indirect application of SECI model.

Compared to other ways to realize organizational way, SECI model could realize the organizational goal and gradual 
dynamic and continuous optimization of the realization effect of the organizational goal through knowledge creation spiral. One prominent feature in SCEI's realization of organizational goal is to stimulate the power of individual and get together the power of collective. Through socialization and externalization processes of knowledge transformation, individual can fully develop his talent and intelligence. Through effective combination of individual through $\mathrm{Ba}$, the close cooperation among individuals can be realized and efforts can be made to realize the same organizational goal [5].

Based on studies on innovative management model and optimization business process of SECI model in many fields such as discipline construction and product $\mathrm{R} \& \mathrm{D}$, the visitors of $\mathrm{HK}$ and Macao colleges and the receiver YUFE are generally regarded as two individuals when applying SECI model to the management model of "Exchange Program" to jointly realize the main goal of the management strategy of the project of "Exchange Program". Therefore, the following aspects will be discussed.

\section{A. The manifestation of the project management of "Exchange Program" in four transformation processes and BAs.}

- Socialization and Originating Ba:

During the implementation of "Exchange Program", socialization is manifested by the direct interaction between the receiver YUFE and visiting $\mathrm{HK}$ and Macao college exchange groups, and observation and movement of visitors. Thus, tacit knowledge is obtained. By virtue of socialization, the reception personnel of the YUFE can feel and know about the inner ideas of visiting groups. Through spreading of such tacit knowledge, a common knowledge transformation $\mathrm{Ba}$ is intangibly created. As the direct and face-to-face contact experience between the receiver and visiting groups is very important to spreading and transformation of tacit knowledge, it is necessary to design an open atmosphere without psychological burden so as to spread tacit knowledge. At this stage, the strategies adopted by the receiver include: to choose the receiving members who has similar background with visiting groups as possible; to give Yunnan fresh flower pie and rose as present on receiving the visiting groups; to hold welcome banquet that both parties attend on the day when the visiting groups arrive, during which small games can be organized so that all can be familiar with each other and trust relationship could be built and a foundation could be laid for creating open and inclusive atmosphere.

\section{- Externalization and Interacting Ba:}

At this stage, as some measures or devices are used to transform the tacit knowledge into the explicit knowledge, the receiver shall actively dominate the process, expressing the ideas or intention of the visitors with written materials. At this Interacting/Dialoguing $\mathrm{Ba}$, the receiver shall exchange with the visiting members over opinions through measures such as setting questionnaire and organizing project seminar. However, the coverage of questionnaire is not comprehensive and the participants of seminar are usually only the receiver. There are few opportunities for knowledge externalization for visiting groups.

- Combination and Systemizing Ba:

For the receiver, the combination indicates the process of integrating the knowledge \& experience of existing "Exchange Program" by combining the external excellent practice into a system. Secondly, the integrated knowledge is expressed again so that both parties can get new inspirations and expand existing knowledge reserve. The Systemizing $\mathrm{Ba}$ at this stage is summarized based on deepening of knowledge \& experience at former two stages, it can be manifested by two aspects:

Firstly, interacting through virtual world and strengthening the transformation of knowledge at this stage through integration of knowledge \& message including network library. The receiver ensures that the related staff can be kept in touch with when problem occurs so that the visiting groups could know about the project content, lecture information and related knowledge of Yunnan as soon as possible. The receiver designs special WeChat electronic manual for visiting groups so that they could browse it on mobile terminal anytime.

Secondly, YUFE has carried out seminar discussion over project line and optimization innovation and nominated the project as "appointment of colorful cloud" since being approved into the "Exchange Program". In implementing the project, YUFE lays emphasis of characteristics and attractions of Yunnan and elaborately designs and integrates 8 investigation paths including "trip to natural resource investigation in Yunnan (Yunnan snub-nosed monkey)", "trip to Wumeng region in Northeast Yunnan", "trip to the handicraft culture of southern Yunnan", "trip to handicraft culture of Lijiang", "trip to handicraft culture of Nan Zhao", "experience of folk customs of Miao ethnic minority of Yunnan", "colorful cloud above spring city" and "trip to public welfare" with the characteristics of Yunnan, focusing on four themes such as "secret area of colorful cloud", "roadway of colorful cloud", "folk customs of colorful cloud" and "public welfare of colorful cloud". YUFE explores ethnic minorities' cultures including Bai, Miao and Yi, covering handicraft experience of porcelain, sculpture and woodcarving, paying visit to Yunnan hope primary school and welfare institutions to start public welfare activities and unique theme contents by paying visit to the surrounding fresh flower origins. The seminar content involves the latest achievements in many fields and disciplines such as economy, politics and culture, thus not only penetrating through the macroscopic content of Chinese business curriculum system but also integrating politics, economy, society and culture of ethnic minorities of Western Yunnan. By organizing a 
series of dynamic and static activities through combination of lecture and field investigation \& theory and practice, the visiting groups have preliminary understandings about economic and social development, cultural background, educational system and reform of ethnic minorities in Western Yunnan. On this basis, the preliminary framework is completed for systematic management operation of the "Exchange Program"

- Internalization and Exercising Ba:

For the dominant party of the "Exchange Program" is the receiver, so the receiver continually practice the knowledge result of the last stage to ultimately internalize these knowledge. Apart from staff in office of HK, Macao and Taiwan, different students will be selected to take part in the program according to the different themes, due to huge mobility of staff, the receiver loses the knowledge during internalization.

To sum up, during organizational management of the "Exchange Program" of YUFE, the program is basically smoothly implemented as there are many strategies for transformation program of "socialization" and "combination". However, there is a lack of useful measures for "externalization" and "internalization".

\section{B. Suggestions for Project Management of "Exchange Program"}

There is deficiency in "externalization" and "internalization" of project management of "Exchange Program". By bringing the unique role of SCEI model into full play in creating individual and organizational knowledge and realizing organizational goal, feasible strategies for improvement are proposed as:

According to the SECI model, the premise of realizing knowledge sharing is that team members express it in specific situation and in the form of collective reflection through continuous dialogue [6]. It is necessary to improve the function of Dialoguing $\mathrm{Ba}$, build regular exchange mechanism with visiting groups, increase opportunities for organizational communication, solve the problems and puzzles occurring to the receiver and visitors in the project and improve their knowledge reserve by sharing experience. As the time for both sides to know about each other is only during the period of implementing the project, it is advised to make up for the deficiency in offline exchanges by virtue of various online media. Meanwhile, the barrier in time and space can be overcome by using socializing tools such as WeChat group to realize more sufficient exchanges and communications of both parties.

Encourage the receiver to gradually summarize experience during the service and record it by taking notes to gain new insights by reviewing the notes, and tap the knowledge and experience of the excellent management staff of the receiver and try to sublimate and save these knowledge. For instance, by regularly holding experience exchange meeting for excellent management staff, the domination and documentation of knowledge and experience of these excellent management staff can be realized, and internal document library can be also built. It can be spread among receivers to increase working skills and experience of the reception staff.

\section{CONCLUSIONS}

YUFE improves the quality of the project of "Exchange Program" and builds diversified filed experience content through innovative and considerate implementation thoughts and by making explorations through experience according to the features of knowledge transformation in SECI model, and realize some organizational goals through knowledge-creation spiral. There is still space for improvement in exchange mechanism and storage and sharing of management knowledge to further improve service level \& quality and create a more diversified exchange platform and space for visiting groups. The "the belt and road" initiative benefits the construction of community of shared future and there is huge development opportunities and potentials. The exchange among colleges in HK, Macao and mainland under the radiation of "the belt and road" is an important driving force for HK future society and educational development. Both sides should jointly deepen the management mechanism of "plan thousands of people" by taking "the Exchange Program" as a fabulous opportunity to realize a win-win situation.

\section{ACKNOWLEDGMENT}

I would like to hereby extend my heartfelt thanks to Professor Katsuhiro Umemoto and Professor Yasunobu Ito, and my colleagues and partners for their considerate support to help me complete the article.

\section{REFERENCES}

[1] M. Polanyi, Study of Man, Chicago: The University of Chicago Press, 1958.

[2] Q.H. Wu, "Knowledge management of Ikjiro Nonaka ", Enterprise Management, vol.5, 2017, pp.18.

[3] I. Nonaka, H. Takeuchi, The Knowledge-Creating Company, New York: Oxford University Press, 1995.

[4] Li J, Z. Zhou, S.J.Zhou, "Example of integration of the east and west: Ikjiro Nonaka and Knowledge Management", Tsinghua Business Review, vol.1-2, 2017, pp.94-95.

[5] Q. Liu, J. Li, "Study on Library Discipline Service Capacity of Colleges Based on SECI Knowledge Creation Spiral”, Library, vol.4, 2017, pp.59.

[6] A.X.Wang, L.Chen, S.Q.Yu, "Design and Application Study of Teacher Training Activities Based on SECI Model", New Ideas and Practice of Teachers Professional Development, vol.10, 2016, pp.26. 